\title{
Effects of iron supplementation twice a week on attention score and haematologic measures in female high school students
}

Akram $\underline{\text { Rezaeian }}{ }^{1}$, MSc, Majid $\underline{\text { Ghayour-Mobarhan }}{ }^{2}$, MD, PhD, Seyed Reza Mazloum ${ }^{3}$, MSc, Mehri $\underline{\text { Yavari }}^{4}$, MSc, Seyed-Ali $\underline{\text { Jafari }}^{5}$, MD

\begin{abstract}
INTRODUCTION Iron deficiency, associated with a decline in cognitive function, is the most common nutritional deficiency globally. The present study aimed to identify the impact of weekly iron supplements on the attention function of female students from a high school in North Khorasan Province, Iran.

METHODS This was a blind, controlled, clinical trial study, involving 200 female students who were chosen using the stratified randomised sampling method. First, laboratory studies were performed to detect iron consumption limitations. Next, the 200 students were divided randomly and equally into case and control groups. The case group was treated with $50 \mathrm{mg}$ of ferrous sulfate twice a week for 16 weeks. We compared both groups' data on attention, iron status and erythrocyte indices. Questionnaires were used to collect demographic data, while clinical data was collected using complete blood count and Toulouse-Piéron tests. Data was analysed using descriptive statistics, as well as paired and independent $t$-tests. RESULTS The mean attention scores of the case and control groups were $104.8 \pm 7.0$ and $52.7 \pm 9.6$, respectively $(p<0.001)$. The mean haemoglobin levels of the two groups were $12.5 \pm 0.9$ and $11.2 \pm 1.0$, respectively $(p<0.001)$. Compared to the control group, the attention scores and haemoglobin concentrations of the case group were found to be improved by approximately $90 \%$ and $10 \%$, respectively.

CONCLUSION Oral iron supplements (50 mg twice a week for 16 weeks) were able to improve the attention span and haematologic indices of female high school students.
\end{abstract}

Keywords: attention, erythrocyte indices, haemog/obin, iron, oral supplement

\section{INTRODUCTION}

Iron deficiency (ID) is the most common nutritional deficiency globally and is especially prevalent in developing countries, with anaemia being the most recognised consequence of the deficiency.$^{(1)}$ In addition to damaged mental function and delayed psychomotor development, anaemia due to ID can result in physical weakness, decreased activity tolerance, impaired physical growth, immune system weakness, body temperature regulation difficulty, changes in energy metabolism, decreased ability to fight infections, and increased mortality. ${ }^{(2)}$ Macroeconomic estimates in less developed countries suggest that anaemia due to ID can be an important factor in the intergenerational transmission of poverty, as it can impact a person's understanding of education and participation in school..$^{(3)}$

Although the most well-known effects of ID are associated with anaemia, we now know that many organs show symptoms of the deficiency before any decrease in blood haemoglobin is observed.(2) ID is more common in girls than boys, largely due to the fact that girls experience menstrual blood loss after menarche. According to the Centers for Disease Control and Prevention, the estimated prevalence of ID in males aged $12-19$ years is less than $1 \%$, while the prevalence in females of the same age range is $11 \%{ }^{(4)}$ In a study by Fakhre-Movahedi and Ahadi, the prevalence of ID in girls living in Iran was reported to be approximately $9.5 \% .{ }^{(5)}$
A feature of the human mental capacity is attention. Attention affects various dimensions of life, including behaviour and personality. ${ }^{(6)}$ As observational studies have shown that ID is associated with a decline in cognitive function, ${ }^{(7)}$ interventional studies aimed at evaluating this relationship have been undertaken. A study by Murray-Kolb and Beard, which investigated the effect of iron treatment, found that young women who received iron supplements for 16 weeks showed significant improvements in their attention, short-term memory, long-term memory and mental performance, even though many of them were not anaemic at the start of the study. ${ }^{(8)}$ The study showed that even moderate levels of ID have a negative effect on the mental function of young women. ${ }^{(8)}$ Bruner et al showed that non-anaemic women with ID who received iron supplements performed better in language comprehension tests than women who received placebos. ${ }^{(7)}$

Based on the meta-analysis by Falkingham et al, only 14 randomised controlled trials (RCTs) involving children aged $>6$ years, adolescents and women were found to have been conducted (up to the year 2010). ${ }^{(9)}$ Of these 14 RCTs, only three cases focused on attention and concentration. ${ }^{(9)}$ This limited number of cases is insufficient to form a basis for any evidencebased decision-making. Furthermore, those studies were done using high doses of iron (i.e. $150 \mathrm{mg}$ per day). ${ }^{(9)}$ As one of the

${ }^{1}$ Scientific Board of Faculty of Nursing and Midwifery, ${ }^{2}$ Biochemistry of Nutritional Research Center, School of Medicine, ${ }^{3}$ Nursing and Midwifery School, ${ }^{4}$ Educational Development Office, Nursing and Midwifery School, ${ }^{5}$ Department of Pediatric Gastroenterology, Mashhad University of Medical Sciences, Mashhad, Iran

Correspondence: Mrs Mehri Yavari, Registrar, Education Development Office, Faculty of Nursing and Midwifery, Mashhad University of Medical Sciences, Ibn Sina Street, Mashhad, Iran. yavarim@mums.ac.ir 
most common problems encountered during iron treatment is gastrointestinal complication, which reduces treatment tolerance and acceptance, ${ }^{(10)}$ the present study aimed to assess the impact of a tolerable dose of iron (i.e. 50 mg twice a week) on the attention function and haematologic indices of female students from a high school in Iran.

\section{METHODS}

One of the four female high schools in Shirvan, Khorasan, Iran, was randomly selected using the simple draw method. As these four female high schools are public schools that enrol students from the general population, no major difference with regard to the socioeconomic statuses of the students from the four schools is expected. We used a simple draw to determine whether the morning or afternoon shift would be assigned as the case group - the morning shift was assigned as the control group, and the afternoon shift, the case group. The morning and afternoon shifts were fixed shifts, with students from both shifts having no formal contact. The students only share the school premises; there were no overlaps in terms of schooling hours and school staff.

Students from the morning and afternoon shifts were divided into four categories according to age group: 14.1-15.0 years, $15.1-16.0$ years, $16.1-17.0$ years and $17.1-18.0$ years. To determine the sample size needed for the present study, the results of our pilot study and the statistical formula for comparing two means (with a confidence coefficient of $95 \%$ and a power of $80 \%$ ) were used. An estimated 63 students per group was required. To ensure that there would be a sufficient number of students in the study, 100 students from each shift (i.e. a total of 200) were selected for participation in the present study. Using systematic random sampling, 25 students were selected from each age group. The value of $k$ used in the present study's systematic random sampling was obtained by dividing the total population of a particular age group (morning or afternoon shifts) by 25 . All 200 students completed the study.

After a brief (i.e. no elaboration regarding outcome measures, such as attention performance and the potential impact of iron on the attention performance) face-to-face explanation of the study, informed consent was obtained from all participants and their parents. All participants completed a researcher-made demographic questionnaire. It includes questions regarding age, age at menarche, maternal age, maternal educational level (in education years), paternal education level (in education years), and number of children in the family. The questionnaire was validated by ten faculty members from the fields of nursing, medicine and statistics. This demographic questionnaire was used to extract data about the students, including their social status. The information was used to statistically control the case and control groups with regard to possible confounding factors.

The Toulouse-Piéron test was used to determine the attention score. This test is one of the most practical standard tests for attention performance. It is a culture-free test composed of several tailed recurrent cubes, with three cubes located at the top of the examination sheet that function as a template. Participants are instructed to search the repeated cubic lines and specify the cubes that are similar to the template cubes within a specific time (i.e. $5 \mathrm{~min}$ ). In order to minimise distractions, each participant completed the test individually in an isolation room with acoustic walls that reduce external noise penetration. The room had constant light, and the shutters of the windows were drawn. Attention score was mathematically calculated (individual attention score $=$ total number of right choices - total number of wrong and forgotten choices). Concurrent validity of this test with the Bourdon distraction test had been reported as $0.74 . .^{(11)}$ In the present study, the validity of this test using the test-retest method was 0.81 .

Complete blood count (CBC) was done to obtain information such as possible contraindications for consumption of iron supplements. Students with high levels of haemoglobin $(\geq 16 \mathrm{~g} / \mathrm{dL})$ and red blood cell count $(\geq 5.03$ million $/ \mu \mathrm{L}$ ) were considered to meet the criterion for iron supplement contraindication. ${ }^{(12)}$ As no contraindication was found among the participants of the present study, none of the students were excluded from the study due to this criterion. Haemoglobin levels were measured using the cyanmethaemoglobin method, while the other parameters were measured using an automated analyser.

Students in the case group, whether anaemic or not, received a $50 \mathrm{mg}$ ferrous sulfate tablet every Wednesday and Saturday for 16 weeks. The conditions of the control group were identical to that of the case group, except for the administration of ferrous sulfate tablets. The CBC and Toulouse-Piéron tests were repeated in the two groups after completion of the treatment programme. The present study did not have a follow-up period and there was no complete sample loss. However, there was some data loss, particularly in terms of $\mathrm{CBC}$ and demographic data; the available data used in the data analysis is reported as degrees of freedom in the tables in the Results section of this manuscript. In cases where CBC parameters were not reported, the analysis was performed based on the available data.

The present study was a blinded study - the person who was responsible for the distribution of the ferrous sulfate tablets differed from the person responsible for conducting the Toulouse-Piéron test. In other words, the examiner did not know whether the individual tested belonged to the case or control group. The person in charge of the data analysis was also not aware whether the data belonged to the control or case group. Although the participants in the case and control groups were aware of whether they had or had not consumed the drug, the nature of the indicators used in the present study were objective (e.g. red blood count indices) or approximately objective (e.g. attention score). Thus, it is unlikely that the participants' awareness on the consumption/lack of consumption of the drug would have had a major effect on the results.

The collected data from both the case and control groups were analysed using descriptive statistics and $t$-tests. To compare the means of normally distributed quantitative variables before and after iron treatment, Kolmogorov-Smirnov and paired sample $t$-tests were used. To compare the means of the case group with that of the control group, independent sample $t$-test was used. Data analysis was done using the Statistical Package for the Social Sciences version 12.0 (SPSS Inc, Chicago, IL, USA). 
Table I. Demographic characteristics of the study population $(n=200)$.

\begin{tabular}{|c|c|c|c|c|c|}
\hline \multirow[t]{2}{*}{ Characteristic } & \multicolumn{3}{|c|}{ Mean \pm SD } & \multicolumn{2}{|c|}{ Independent $t$-test } \\
\hline & Case group & Control group & Total & df & p-value \\
\hline Age (yr) & $16.10 \pm 1.40$ & $16.44 \pm 1.23$ & $16.20 \pm 1.30$ & 198 & 0.084 \\
\hline Age at menarche $(\mathrm{yr})$ & $13.46 \pm 1.03$ & $13.63 \pm 1.12$ & $13.50 \pm 1.00$ & 198 & 0.273 \\
\hline Maternal age (yr) & $41.14 \pm 5.23$ & $40.13 \pm 3.29$ & $40.70 \pm 4.38$ & 196 & 0.103 \\
\hline Maternal education* & $5.70 \pm 5.02$ & $6.80 \pm 5.65$ & $6.20 \pm 5.30$ & 196 & 0.129 \\
\hline Paternal education* & $6.74 \pm 5.43$ & $7.63 \pm 5.75$ & $7.10 \pm 5.60$ & 196 & 0.272 \\
\hline No. of children in the family & $5.20 \pm 2.05$ & $4.65 \pm 2.13$ & $4.90 \pm 2.10$ & 196 & 0.068 \\
\hline
\end{tabular}

* Measured as number of years of education. df: degrees of freedom; SD: standard deviation

The present study was approved by the ethics committee of the Islamic Azad University of Bojnourd, Iran. Participation from students in the present study was voluntary. For ethical considerations, participants were informed about the need for blood sampling, the amount of blood to be taken, possible blood sampling complications, the tests that would be performed on the blood drawn, the name of the drug administered, the possible benefits and side effects of the drug (only in the case group), and the privacy of the obtained information. After the information was given, the students and their parents signed informed consent forms. The consent forms were kept until the completion of the study. Upon completion of the present study, the laboratory results and the results of the Toulouse-Piéron test were returned to the students. In a final meeting, the results of the study were communicated to all participants (i.e. both the case and control groups). Students who still had haemoglobin levels $<12 \mathrm{~g} / \mathrm{dL}$ on the second $\mathrm{CBC}$ were referred to specialists for further evaluation and treatment.

\section{RESULTS}

The mean age at menarche and mean age of the study population $(\mathrm{n}=200)$ were $13.5 \pm 1.0$ years and $16.2 \pm 1.3$ years, respectively. Of the 200 girls, $20.6 \%$ had haemoglobin levels $<12 \mathrm{~g} / \mathrm{dL}$. The case and control groups did not show any statistically significant difference in their demographic characteristics (i.e. age, age at menarche, maternal age, maternal educational level, paternal education level and number of children in the family) (Table I). The two groups also did not show any statistically significant difference in the pre-intervention measures (i.e. the attention score based on the Toulouse-Piéron test and the CBC parameters).

The post-intervention attention score of the case group was significantly higher than that of the control group $(p<0.001)$ (Table II). There was also a significant difference between the mean haemoglobin $(p<0.001)$, haematocrit $(p<0.001)$ and corpuscular haemoglobin $(p=0.012$ ) levels of the two groups after intervention. Based on the comparisons done within the case group using paired sample $t$-test, the post-intervention attention score was significantly higher than the pre-intervention score $(p<0.001)$. Within the control group, this difference was not statistically significant. Differences in mean haemoglobin, haematocrit and corpuscular haemoglobin levels before and after intervention were also statistically significant in the case group, with the levels of the three aforementioned mean values increased after intervention. In the control group, those mean values were significantly lower after intervention. Both $\mathrm{CBC}$ and mean corpuscular volume were also significantly increased in the case group after intervention. In the control group, however, no significant differences between pre- and post-intervention values (i.e. $\mathrm{CBC}$ and mean corpuscular volume) were observed. No significant difference in mean corpuscular haemoglobin concentration was observed between the two study groups.

Changes were observed before and after intervention. The changes seen in the case group were significantly greater than those seen in the control group. In the case group, the greatest and lowest changes were related to attention score and mean corpuscular haemoglobin concentration, respectively (Table III). The correlation between attention score and the haemoglobin levels at the post-intervention stage was statistically, but not strongly, significant $(r=0.506, p<0.01)$. Table III shows the change in the variables at baseline and after intervention (in percentage); the changes are significant for all measured variables.

\section{DISCUSSION}

The results of the present study provide evidence for the positive effects of iron supplementation twice a week on the attention performance and haematologic measures of high school female students. The case and control groups did not show any statistically significant difference in their demographic characteristics (i.e. age, age at menarche, maternal age, maternal educational level, paternal education level and number of children in the family). This is important, as these characteristics are known to be associated with ID. Age is a factor involved in the creation and manifestation of anaemia, ${ }^{(12)}$ and also affects the quality of a person's attention. ${ }^{(13)}$ Other than age, menstruation is a risk factor for the development of anaemia, ${ }^{(14)}$ with decreased age at menarche associated with an increased risk of ID. ${ }^{(15)}$ Maternal age and education level may affect the educational opportunities of female students, ${ }^{(14)}$ and as previous studies have shown, poverty is a risk factor for ID. ${ }^{(16)}$ Paternal education level, to some extent, could affect family income, and thus, nutrition. ${ }^{(17)}$ The number of children in a family is a criterion that can be affected by poverty, and can also affect the nutritional opportunities of the family members. ${ }^{(18)}$

Data analysis showed that the difference in mean attention scores between the case and control groups was statistically significant $(p<0.001)$. Comparisons done within the case group, before and after iron supplementation, showed that the difference in the attention scores was statistically significant $(p<0.001)$. The 
Table II. Results of the Toulouse-Piéron and complete blood cell tests before and after intervention.

\begin{tabular}{|c|c|c|c|c|}
\hline \multirow[t]{2}{*}{ Variable } & \multicolumn{2}{|c|}{ Mean \pm SD } & \multicolumn{2}{|c|}{ Independent $t$-test } \\
\hline & Case group & Control group & df & p-value \\
\hline \multicolumn{5}{|l|}{ Attention score } \\
\hline Post-intervention & $104.80 \pm 7.0$ & $52.70 \pm 9.6$ & 198 & 0.001 \\
\hline Paired sample $t$-test & $\mathrm{df}=99 ; \mathrm{p}<0.001$ & $d f=99 ; p=0.122$ & & \\
\hline \multicolumn{5}{|l|}{ Haemoglobin (g/dL) } \\
\hline Post-intervention & $12.50 \pm 0.97$ & $11.20 \pm 1.00$ & 198 & $<0.001$ \\
\hline Paired sample $t$-test & $d f=99 ; p<0.001$ & $d f=99 ; p<0.001$ & & \\
\hline \multicolumn{5}{|l|}{ Haematocrit (\%) } \\
\hline Pre-intervention & $33.90 \pm 3.3$ & $34.40 \pm 3.5$ & 198 & 0.224 \\
\hline Post-intervention & $37.40 \pm 2.9$ & $33.60 \pm 3.1$ & 198 & $<0.001$ \\
\hline Paired sample $t$-test & $d f=99 ; p<0.001$ & $d f=99 ; p<0.001$ & & \\
\hline Pre-intervention & $80.90 \pm 1.9$ & $81.20 \pm 2.4$ & 198 & 0.427 \\
\hline Post-intervention & $81.30 \pm 1.9$ & $81.10 \pm 2.4$ & 198 & 0.563 \\
\hline Paired sample $t$-test & $d f=99 ; p<0.001$ & $d f=99 ; p=0.132$ & & \\
\hline \multicolumn{5}{|l|}{ MCH (pg/cell) } \\
\hline Pre-intervention & $30.20 \pm 1.6$ & $30.50 \pm 1.5$ & 198 & 0.140 \\
\hline Post-intervention & $30.90 \pm 1.9$ & $30.30 \pm 1.3$ & 198 & 0.012 \\
\hline Paired sample $t$-test & $d f=99 ; p=0.032$ & $d f=99 ; p=0.004$ & & \\
\hline \multicolumn{5}{|l|}{ MCHC (g/dL) } \\
\hline Pre-intervention & $32.20 \pm 0.63$ & $32.40 \pm 0.60$ & 198 & 0.063 \\
\hline Post-intervention & $32.30 \pm 0.67$ & $32.30 \pm 0.78$ & 196 & 0.780 \\
\hline Paired sample $t$-test & $d f=99 ; p=0.144$ & $d f=99 ; p=0.083$ & & \\
\hline
\end{tabular}

df: degrees of freedom; $\mathrm{MCH}$ : mean corpuscular haemoglobin; $\mathrm{MCHC}$ : mean corpuscular haemoglobin concentration; MCV: mean corpuscular volume; SD: standard deviation

Table III. Changes in the measured variables before and after intervention.

\begin{tabular}{lrrrr}
\hline Variable & \multicolumn{2}{c}{ Change (\%) } & df & p-value \\
\cline { 2 - 3 } & $\begin{array}{c}\text { Case } \\
\text { group }\end{array}$ & $\begin{array}{c}\text { Control } \\
\text { group }\end{array}$ & & \\
\hline Attention score & 98.50 & 3.40 & 198 & $<0.001$ \\
Haemoglobin (g/dL) & 10.70 & -2.60 & 198 & $<0.001$ \\
Haematocrit (\%) & 10.60 & -2.60 & 198 & $<0.001$ \\
MCH (pg/cell) & 2.30 & -0.65 & 198 & $<0.001$ \\
MCV (fL) & 0.49 & -0.06 & 198 & $<0.001$ \\
MCHC (g/dL) & 0.15 & -0.21 & 198 & 0.015 \\
\hline
\end{tabular}

$\mathrm{df}$ : degrees of freedom; $\mathrm{MCH}$ : mean corpuscular haemoglobin; $\mathrm{MCHC}$ : mean corpuscular haemoglobin concentration; MCV: mean corpuscular volume

attention scores of the female students who received $50 \mathrm{mg}$ of ferrous sulfate twice a week for 16 weeks were higher after iron supplementation, as compared to their scores before treatment. The scores were also higher than that in the control group.

In the control group, we observed a post-intervention decrease in CBC parameters, such as haemoglobin levels, haematocrit levels, mean corpuscular volume, mean corpuscular haemoglobin and mean corpuscular haemoglobin concentration, from the baseline levels. However, the same CBC parameters were higher than the baseline levels in the case group. This indicates that the amount of iron in the diet of the female students in the control group was not sufficient to meet growth requirements and offset the disposal of blood via menstruation.

The change in the scores of the Toulouse-Piéron test in both the case and control groups was also approximately parallel to the change in the results of the $\mathrm{CBC}$ parameters. The attention score increased in both groups after intervention, but the increase was significantly greater in the case group. In the control group, the increase observed in this parameter could be due to the mere-exposure effect. In the present study, we controlled for this effect by making changes to the post-intervention examination sheet; the recurring pattern of the cubes and the template at the top of the examination sheet were different from that in the preintervention examination sheet. However, this effect cannot be eliminated completely. ${ }^{(19)}$

From the results of the present study, the consumption of $50 \mathrm{mg}$ ferrous sulfate tablets twice a week for 16 weeks significantly improved both the attention performance and haemoglobin levels of the students in the case group. However, while the attention performance of the case group improved by $98 \%$ compared to the baseline, the haemoglobin levels of the case group increased by only $10 \%$ compared to the baseline levels. If we factor in the $3 \%$ attention performance increase (attributable to the mere-exposure effect) seen in the control group, the postintervention attention performance increase in the case group would be about $95 \%$ from the baseline. Iron can affect attention performance through neurotransmitter pathways, haemoglobin production and brain oxygenation. ${ }^{(7)}$ Based on the $10 \%$ and 95\% improvements in the haemoglobin levels and attention performance of the case group, respectively, we postulate that the iron supplementation in the present study affected attention performance mainly via neurotransmitter pathways rather than via haemoglobin production. The significant, albeit relatively weak, correlation between haemoglobin levels and attention 
score supports this point. Previous studies have also shown that cognitive signs of ID are reported before significant decline in haemoglobin levels. ${ }^{(8)}$

Although the effects of ID on cognitive outcomes in childhood have been studied in detail, evidence for the association between ID and cognitive outcomes in adults are limited. The effects of iron supplementation on the attention/concentration of anaemic and iron-deficient participants have been studied. Iron supplementation appears to have statistically significant beneficial effects on overall attention/concentration in both anaemic and iron-deficient participants, without any suggestion of heterogeneity. ${ }^{(9)}$ Falkingham et al interpreted this effect as a 'moderate effect'. ${ }^{(9)}$ According to the review and meta-analysis conducted by Falkingham et al, improvement in attention/concentration was found to have occurred in the set of studies involving children aged 6-18 years, with no suggestion of heterogeneity. However, the only study on premenopausal women did not suggest a statistically significant effect. Nonetheless, Falkingham et al's study addressed the positive effects of iron supplementation on the cognitive performance of older children. The study population and results reported are similar to that of the present study, thereby supporting the results of the present study.

In 2007, Murray-Kolb and Beard examined the relationship between iron status and the cognitive abilities in young women. ${ }^{(8)}$ After treatment, a significant improvement in serum ferritin was associated with a 5-7-fold improvement in cognitive performance, whereas a significant improvement in haemoglobin was related to improved speed in completing cognitive tasks. ${ }^{(8)}$ Although the participants in the case group of the present study were not selected based on the presence of anaemia, the mean haemoglobin level of the participants before the intervention was $11.30 \pm 1.13 \mathrm{~g} / \mathrm{dL}$, which is within the anaemic range for their age group. After intervention, their mean haemoglobin level was $12.50 \pm 0.97 \mathrm{~g} / \mathrm{dL}$, which is above the anaemic range for their age group. Thus, it can be posited that the significant increase in the attention score of the case group after iron treatment (as compared to the case group before iron supplementation, and as compared to the control group) could be associated with the increase in the haemoglobin levels. Concurring with the results of the study by Murray-Kolb and Beard, ${ }^{(8)}$ this association seen in our present study may be a result of the direct effect increased serum iron has on the brain's processing performance.

Although various studies have supported the positive effects of iron supplementation on cognitive performance, ${ }^{(7,20)}$ there are recent studies that consider daily iron supplementation to result in an excessive source of iron supply. ${ }^{(10)}$ Additionally, the adverse effects of iron supplementation, especially in high doses, may result in diminished patient compliance. ${ }^{(21)}$ Therefore, the current approach is to replace daily doses with weekly doses, especially since some studies have shown that the benefits of weekly doses are greater than those of daily doses. ${ }^{(21)}$ For example, a study by Khalid et al compared the effectiveness of daily iron treatment with the effectiveness of weekly iron treatment via a randomised longitudinal study. ${ }^{(21)}$ The authors found that other than an increased likelihood of compliance with the use of weekly doses, criteria such as haematocrit levels, red blood cell count and reticulocyte count were better in the group receiving weekly doses, as compared to the group receiving daily doses. ${ }^{(21)}$ In the present study where an iron dose of $100 \mathrm{mg}$ per week was used, haemoglobin and red blood cell indices were improved in the case group when compared to the control group. Although the dose of iron used in the present study was half of that used in the study by Khalid et al, ${ }^{(21)}$ we obtained similar results. This was expected as the study population of the present study was different from the study population of the study by Khalid et al their study population consisted of pregnant women who required an increased iron intake due to fetal growth. ${ }^{(21)}$ In the present study, our study population consisted of pubescent female students who had an increased iron requirement due to the physiological changes that came with puberty (i.e. monthly blood loss). Iron requirement during puberty is two times the normal requirement, ${ }^{(22)}$ whereas in pregnancy, it is four times the normal requirement. ${ }^{(20)}$ It is therefore rational that although the dose used in the present study was half of that used by Khalid et al, ${ }^{(21)}$ similar results were achieved. In other populations, similar results have been achieved with lower doses of iron. Schultink et al compared the effect of $30 \mathrm{mg}$ of iron daily with $30 \mathrm{mg}$ of iron twice a week and concluded that in preschool children with low iron status, the effect that iron supplementation twice a week has on iron status is similar to that of the effect daily supplementation has on iron status. ${ }^{(10)}$

The present study is unique in terms of its study population and dose of iron supplementation. However, one limitation is the lack of a placebo control. However, as the outcome measures were objective (i.e. CBC measures) and semi-objective (i.e. attention score), the probability of the results being affected by the mental impressions of the participants is minimal.

In conclusion, based on the results of the present study and of previous studies, there is evidence that weekly iron supplementation has considerable benefits with regard to treating anaemia, elevating serum iron, improving cognitive function, and improving patient compliance with treatment regimen. Well-powered, independently funded, blinded, placebo-controlled RCTs of at least one year's duration and involving participants of different age groups (including children, adolescents, adults and older people, and people across all levels of baseline iron status) are required in order to validate the findings of the present study. Also, as iron supplementation resulted in a positive effect on the attention performance of students in the present study, the effect of iron supplementation on student achievement could be an interesting area for further research.

\section{ACKNOWLEDGEMENT}

This study was approved and financially supported by the Vice Chancellor of the Islamic Azad University of Bojnourd, Iran.

\section{REFERENCES}

1. Lutter CK. Iron deficiency in young children in low-income countries and new approaches for its prevention. J Nutr 2008; 138:2523-8.

2. Behrman S, Kliegman R. Barbara J. Nelson Textbook of Pediatrics. 19th ed. Philadelphia: Saunders, 2011. 
3. Bobonis G), Miguel E, Puri-Sharma C. Anemia and school participation. J Human Resource 2006; 41:692-721.

4. Recommendations to prevent and control iron deficiency in the United States. Centers for Disease Control and Prevention. MMWR Recomm Rep 1998; 47:1-29.

5. Fakhre-Movahedi A, Ahadi F. Prevalence of iron deficiency anemia in high school girls in Semnan City. JSSU 2005; 12:51-5.

6. Hill SE. DelPriore DJ. Vaughan PW. The cognitive consequences of envy: attention, memory, and self-regulatory depletion. J Pers Soc Psychol 2011; 101:653-66.

7. Bruner AB, Joffe A, Duggan AK, Casella JF, Brandt J. Randomised study of cognitive effects of iron supplementation in non-anaemic iron-deficient adolescent girls. Lancet 1996; 348:992-6.

8. Murray-Kolb LE, Beard JL. Iron treatment normalizes cognitive functioning in young women. Am J Clin Nutr 2007; 85:778-87.

9. Falkingham $M$, Abdelhamid $A$, Curtis $P$, et al. The effects of oral iron supplementation on cognition in older children and adults: a systematic review and meta-analysis. Nutr J 2010; 9:4.

10. Schultink W, Gross R, Gliwitzki M, Karyadi D, Matulessi P. Effect of daily vs twice weekly iron supplementation in Indonesian preschool children with low iron status. Am J Clin Nutr 1995; 61:111-5.

11. Iravani M. Empirical Psychology. 4th ed. Tehran: Arvin Vijeh, 2008.

12. O'Donnell A, Premawardhena A, Arambepola M, et al. Age-related changes in adaptation to severe anaemia in childhood in developing countries. Proc Natl Acad Sci U S A 2007; 104:9440-4.

13. Morton JB, Bosma R, Ansari D. Age-related changes in brain activation associated with dimensional shifts of attention: an fMRI study. Neuroimage 2009; 46:249-56.

14. Wu AC, Lesperance L, Bernstein $\mathrm{H}$. Screening for iron deficiency. Pediatr Rev 2002; 23:171-8.

15. Frith-Terhune AL, Cogswell ME, Khan LK, Will JC, Ramakrishnan U. Iron deficiency anaemia: higher prevalence in Mexican American than in non-Hispanic white females in the third National Health and Nutrition Examination Survey, 1988-1994. Am J Clin Nutr 2000; 72:963-8.

16. Hatton TJ, Martin RM. The Effects on Stature of Poverty, Family Size and Birth Order: British Children in the 1930s. IZA Discussion Papers 3314, Institute for the Study of Labor (IZA). 2008.

17. Moestue $\mathrm{H}$, Huttly $\mathrm{S}$. Adult education and child nutrition: the role of family and community. J Epidemiol Community Health 2008; 62:153-9.

18. Ajao KO, Ojofeitimi EO, Adebayo AA, Fatusi AO. Afolabi OT. Influence of family size, household food security status, and child care practices on the nutritional status of under-five children in Ile-Ife, Nigeria. Afr J Reprod Health 2010; 14:117-26.

19. Ishii K. Does mere exposure enhance positive evaluation, independent of stimulus recognition? A replication study in Japan and the USA. Jpn Psychol Res 2005; 47:280-5.

20. Symonds ME, Ramsay MM, eds. Maternal-Fetal Nutrition During Pregnancy and Lactation. 4th ed. Cambridge: Cambridge University Press, 2010.

21. Khalid S, Ahmad SI, Waqar S, Faisal M. Effectiveness of weekly iron supplementation in anemia in pregnancy. Pak J Pharmacol 2011; 28:9-16.

22. Samour P, King K. Pediatric Nutrition. 4th ed. New York: Jones \& Bartlett Learning, 2010. 